PROCEEDINGS OF THE

AMERICAN MATHEMATICAL SOCIETY

Volume 129, Number 4, Pages 947-954

S 0002-9939(00)05654- 9

Article electronically published on October 10, 2000

\title{
EVERY $\Sigma$-CS-MODULE HAS AN INDECOMPOSABLE DECOMPOSITION
}

JOSÉ L. GÓMEZ PARDO AND PEDRO A. GUIL ASENSIO

(Communicated by Ken Goodearl)

\begin{abstract}
We show that every $\Sigma$-CS module is a direct sum of uniform modules, thus solving an open problem posed in 1994 by Dung, Huynh, Smith and Wisbauer. With the help of this result we also answer several other questions related to indecomposable decompositions of CS-modules.
\end{abstract}

\section{INTRODUCTION}

In recent years there has been considerable interest in the study of CS-modules (also called extending modules), a natural generalization of (quasi-) injective and (quasi-) continuous modules obtained by requiring only that each submodule be essential in a direct summand. In particular, a lot of attention has been devoted to the study of indecomposable decompositions of CS-modules, as can be seen, for example, in [1, 3, 4, 15, 6, 7, 8, 9, 13, 15, 16, 18.

It is well known that a CS-module need not have an indecomposable decomposition and, in fact, every CS right $R$-module has an indecomposable decomposition if and only if $R$ is a right noetherian ring [15]. Thus the study of indecomposable decompositions has been focused mainly on $\Sigma$-CS-modules, i.e., modules $M$ such that every direct sum of copies of $M$ is a CS-module. One reason for this is the fact that the indecomposable decompositions of $\Sigma$-CS-modules are particulary well-behaved. Every indecomposable CS-module is uniform, and every uniform $\Sigma$ CS-module is $\Sigma$-quasi-injective by [1], so that if $M$ is $\Sigma$-CS and $M=\bigoplus_{I} M_{i}$ is an indecomposable decomposition of $M$, then the $M_{i}$ have local endomorphism rings and, moreover, the decomposition complements direct summands by [8]. Thus this decomposition has all the desirable properties that one would expect and, among them, uniqueness as a consequence of the Krull-Remak-Schmidt-Azumaya theorem. But the strongest motivation to study decompositions of $\Sigma$-CS-modules is that, as suggested by the injective case, one expects that indecomposable decompositions are much more abundant for $\Sigma$-CS-modules than for CS-modules. The main open problem in this direction was posed by Dung, Huynh, Smith, and Wisbauer in 9, p. 104], and asks whether every $\Sigma$-CS-module has an indecomposable decomposition. As it was observed in [1 pp. 2378-2379] this problem is equivalent to the question,

Received by the editors April 2, 1999 and, in revised form, July 8, 1999.

1991 Mathematics Subject Classification. Primary 16D70; Secondary 16D50.

This work was partially supported by the DGES(PB96-0961, Spain). The second author was also partially supported by the Fundación Séneca (PB16FS97).

(C)2000 American Mathematical Society 
asked in 7, Remark 3.4], of whether the quasi-injective hull of a $\Sigma$-CS-module is $\Sigma$-quasi-injective.

The purpose of this paper is to settle this problem. We give an affirmative answer in Theorem [2.3, where we show that every $\Sigma$-CS-module is a direct sum of uniform modules. Thus $\Sigma$-CS-modules behave, as far as indecomposable decompositions are concerned, in a way similar to $\Sigma$-injective modules. But this should not lead to the conclusion that everything works just the same in this more general setting. From a technical standpoint we remark that our proof is quite different from the proof for $\Sigma$-injective modules and, on the other hand, the parallelism with injectivity soon breaks down when one looks at $\Sigma$-CS-modules from a more global perspective. For example, it is well known that the modules $M$ such that a countable direct sum of copies of $M$ is CS need not have an indecomposable decomposition. Moreover, it follows from our result that if every CS right $R$-module is $\Sigma$-CS, then $R$ is a right noetherian ring. However, in contrast with the injective case, the converse is far from being true for, as it has been observed by Al-attas and Vanaja in [1, there are even artinian right serial rings such that not every right CS-module is $\Sigma$-CS.

On the way to prove our main theorem, we show that a stronger result holds for CS-modules which have an essential cyclic submodule. We prove in Theorem 2.2 that for these modules it is enough to suppose that an uncountable direct sum of copies of the module is CS to conclude that the module is a direct sum of uniforms. This also applies to all countably generated CS-modules, because they are direct sums of modules which have an essential cyclic submodule. As a consequence of this result we give an affirmative answer to a question posed in [3] by showing that, for a right nonsingular ring $R$, if every $\aleph_{1}$-generated nonsingular right $R$-module is projective, then every nonsingular right $R$-module is projective, which provides a stronger form of a theorem of Goodearl [12, Theorem 2.15].

Throughout this paper all rings $R$ will be associative and with identity, and Mod- $R$ will denote the category of right $R$-modules. By a module we will usually mean a right $R$-module. We refer to [2, 19] for all undefined notions used in the text.

\section{Results}

Recall that a submodule $K$ of an $R$-module $M$ is said to be closed (in $M$ ) when $K$ has no proper essential extensions in $M$. If $L \subseteq M$, then a closed submodule $K$ of $M$ that contains $L$ as an essential submodule (we then write $L \subseteq_{e} K$ ) is called an essential closure of $L$ in $M$. The module $M$ is called CS (or an extending module, cf. 9]) if every closed submodule is a direct summand.

A set $\left\{L_{j} \mid j \in J\right\}$ of independent submodules of $M$ is called a local direct summand of $M$ (cf. [9, p. 66]) when $\bigoplus_{j \in F} L_{j}$ is a direct summand of $M$ for every finite subset $F \subseteq J$. If $\bigoplus_{j \in J} L_{j}$ is a direct summand of $M$, then we will also say that the local direct summand $\left\{L_{j} \mid j \in J\right\}$ is a summand of $M$. We will denote by $|X|$ the cardinality of a set $X$.

The following lemma will play a fundamental role in the proof of our main result. The counting argument we use is inspired by an idea of Osofsky [17].

Lemma 2.1. Let $M$ be a $C S$-module and $\left\{L_{i} \mid i \in I\right\}$ a local direct summand such that $|I|=\aleph_{0}$. Then there exist subsets $\mathcal{A}, \mathcal{K} \subseteq 2^{I}$ such that:

(i) $\mathcal{A}$ is a partition of $I$ with $|\mathcal{A}|=\aleph_{0}$ and $|A|=\aleph_{0}$ for every $A \in \mathcal{A}$.

(ii) $\mathcal{A} \subseteq \mathcal{K},|\mathcal{K}|=\aleph_{1}$ and $|K|=\aleph_{0}$ for each $K \in \mathcal{K}$. 
(iii) $K \cap K^{\prime}$ is a finite set for all $K, K^{\prime} \in \mathcal{K}$ such that $K \neq K^{\prime}$.

(iv) If $L_{K}$ denotes an essential closure of $\bigoplus_{K} L_{i}$ in $M$ for each $K \in \mathcal{K}$, and $K, K_{1}, \ldots, K_{r}$ are pairwise distinct elements of $\mathcal{K}$, then

$$
L_{K} \cap\left(L_{K_{1}}+\cdots+L_{K_{r}}\right)=\bigoplus_{K \cap\left(K_{1} \cup \ldots \cup K_{r}\right)} L_{i} .
$$

Proof. Let $\mathcal{A} \subseteq 2^{I}$ be an infinite partition of $I$ consisting of infinite sets. Using Zorn's lemma, consider a set $\mathcal{B} \subseteq 2^{I}$ which is maximal with respect to the following properties:

(a) $\mathcal{A} \subseteq \mathcal{B}$, (b) $|K|=\aleph_{0}$ for each $K \in \mathcal{B}$, (c) $K \cap K^{\prime}$ is finite for each $K, K^{\prime} \in \mathcal{B}$ such that $K \neq K^{\prime}$.

As in the proof of [11, Lemma 3.1], we see that $|\mathcal{B}|>\aleph_{0}$, and if we take $\mathcal{K}$ to be any subset of $\mathcal{B}$ containing $\mathcal{A}$ and such that $|\mathcal{K}|=\aleph_{1}$, we have that the conditions stated in (i), (iii), and (iii) are fulfilled. Now, for each $K \in \mathcal{K}$, choose an essential closure $L_{K}$ of $\bigoplus_{K} L_{i}$ in $M$. It only remains to prove that $L_{K} \cap\left(L_{K_{1}}+\cdots+L_{K_{r}}\right)=$ $\bigoplus_{K \cap\left(K_{1} \cup \ldots \cup K_{r}\right)} L_{i}$ provided that $K, K_{1}, \ldots, K_{r}$ are pairwise distinct elements of $\mathcal{K}$.

We start by showing that $L_{K} \cap\left(\bigoplus_{\cup_{n=1}^{r} K_{n}} L_{i}\right)=\bigoplus_{K \cap\left(\cup_{n=1}^{r} K_{n}\right)} L_{i}$. Set $N=$ $\bigoplus_{K \cap\left(\bigcup_{n=1}^{r} K_{n}\right)} L_{i}$ and write $\bigoplus_{\bigcup_{n=1}^{r} K_{n}} L_{i}=N \oplus\left(\bigoplus_{\left(\bigcup_{n=1}^{r} K_{n}\right)-K} L_{i}\right)$. Then $N \subseteq L_{K}$ and so, using modularity and bearing in mind that $L_{K} \cap\left(\bigoplus_{\left(\cup_{n=1}^{r} K_{n}\right)-K} L_{i}\right)=0$, we obtain that $L_{K} \cap\left(\bigoplus_{\bigcup_{n=1}^{r} K_{n}} L_{i}\right)=N$. Next, set $X=L_{K} \cap\left(L_{K_{1}}+\cdots+L_{K_{r}}\right)$. We want to show that $X=N$. It is clear that $N \subseteq X$ and, moreover, $N$ is a direct summand of $M$, and hence of $X$, because $K \cap\left(K_{1} \cup \cdots \cup K_{r}\right)$ is a finite set. Thus we can consider a submodule $Z$ of $X$ such that $X=N \oplus Z$ and to complete the proof we must show that $Z=0$. In order to do this, we first write the sum $L_{K_{1}}+\cdots+L_{K_{r}}$ in a special way. Let $H_{1}=0$ and, for each $n=2, \ldots, r$, let $K^{n}=K_{n} \cap\left(\bigcup_{j=1}^{n-1} K_{j}\right)$ and $H_{n}=\bigoplus_{K^{n}} L_{i}$. Again, the fact that $\left\{L_{i}\right\}_{I}$ is a local direct summand implies that $H_{n}$ is a direct summand of $M$ and, since $H_{n} \subseteq L_{K_{n}}$, we can choose a supplement $Y_{n}$ of $H_{n}$ in $L_{K_{n}}$ so that, for each $n=1, \ldots, r$, we have $L_{K_{n}}=Y_{n} \oplus H_{n}$. Observe that $H_{n} \subseteq \sum_{j=1}^{n-1} L_{K_{j}}$ for $n>1$ and so $L_{K_{1}}+\cdots+L_{K_{r}}=Y_{1}+\cdots+Y_{r}$. Now, suppose that $Z \neq 0$, pick an element $0 \neq z \in Z$ and write $z=y_{1}+\cdots+y_{r}$, with $y_{n} \in Y_{n}$. Inductively we construct a sequence of elements $r_{1}, \ldots, r_{n}, \ldots \in R$ such that for each $n \leq r$ we have that $r_{n} r_{n-1} \ldots r_{1} y_{j} \in \bigoplus_{K_{j}} L_{i}$ for each $j=1, \ldots, n$, and

$$
r_{n} r_{n-1} \ldots r_{1} z \neq r_{n} r_{n-1} \ldots r_{1} y_{1}+r_{n} r_{n-1} \ldots r_{1} y_{2}+\cdots+r_{n} r_{n-1} \ldots r_{1} y_{n} .
$$

We define $r_{1}$ in the following way. If $y_{1}=0$, we take $r_{1}=1$ and we have that $r_{1} z=z \neq 0=r_{1} y_{1} \in \bigoplus_{K_{1}} L_{i}$. If $y_{1} \neq 0$, then since $\bigoplus_{K_{1}} L_{i}$ is essential in $L_{K_{1}}$ and $y_{1} \in Y_{1} \subseteq L_{K_{1}}$, we can take $r_{1} \in R$ such that $0 \neq r_{1} y_{1} \in \bigoplus_{K_{1}} L_{i}$. Suppose that $r_{1} z=r_{1} y_{1}$. Then we have that $r_{1} z=r_{1} y_{1} \in L_{K} \cap\left(\bigoplus_{K_{1}} L_{i}\right) \subseteq$ $N$. Hence $r_{1} z \in Z \cap N=0$ and so $r_{1} y_{1}=r_{1} z=0$, a contradiction. Thus $r_{1} z \neq r_{1} y_{1}$. Suppose now that, for $n \leq r$, we have defined $r_{1}, \ldots, r_{n-1} \in R$ such that $r_{n-1} r_{n-2} \ldots r_{1} y_{j} \in \bigoplus_{K_{j}} L_{i}$ for $j=1, \ldots, n-1$, and $r_{n-1} r_{n-2} \ldots r_{1} z \neq$ $r_{n-1} r_{n-2} \ldots r_{1} y_{1}+\cdots+r_{n-1} r_{n-2} \ldots r_{1} y_{n-1}$. Then if $r_{n-1} r_{n-2} \ldots r_{1} y_{n}=0$ we define $r_{n}=1$ and in this case clearly we have that $r_{n} r_{n-1} \ldots r_{1} y_{j} \in \bigoplus_{K_{j}} L_{i}$ for each $j=1, \ldots, n$, and $r_{n} r_{n-1} \ldots r_{1} z \neq r_{n} r_{n-1} \ldots r_{1} y_{1}+\cdots+r_{n} r_{n-1} \ldots r_{1} y_{n}$. On the other hand, if $r_{n-1} r_{n-2} \ldots r_{1} y_{n} \neq 0$, then we take $r_{n}$ to be an element of $R$ such that $0 \neq r_{n} r_{n-1} \ldots r_{1} y_{n} \in \bigoplus_{K_{n}} L_{i}$ so that, again, we have $r_{n} r_{n-1} \cdots+r_{1} y_{j} \in \bigoplus_{K_{j}} L_{i}$ for each $j=1, \ldots, n$. Now, suppose that $r_{n} r_{n-1} \ldots r_{1} z=r_{n} r_{n-1} \ldots r_{1} y_{1}+\cdots+$ 
$r_{n} r_{n-1} \ldots r_{1} y_{n}$. Then $r_{n} r_{n-1} \ldots r_{1} z \in L_{K} \cap\left(\bigoplus_{\bigcup_{j=1}^{n} K_{j}} L_{i}\right) \subseteq N$ and consequently $r_{n} r_{n-1} \ldots r_{1} z \in N \cap Z=0$. Hence $0 \neq r_{n} r_{n-1} \ldots r_{1} y_{n}=-r_{n} r_{n-1} \ldots r_{1} y_{1}-\cdots-$ $r_{n} r_{n-1} \ldots r_{1} y_{n-1} \in\left(\bigoplus_{K_{n}} L_{i}\right) \cap\left(\bigoplus_{\bigcup_{j=1}^{n-1} K_{j}} L_{i}\right)=H_{n}$. Therefore, $r_{n} r_{n-1} \ldots r_{1} y_{n} \in$ $H_{n} \cap Y_{n}=0$, a contradiction. Thus we have $r_{1}, \ldots, r_{n} \in R$ such that $r_{n} r_{n-1} \ldots r_{1} z \neq$ $r_{n} r_{n-1} \ldots r_{1} y_{1}+r_{n} r_{n-1} \ldots r_{1} y_{2}+\cdots+r_{n} r_{n-1} \ldots r_{1} y_{n}$. For $n=r$ we obtain a contradiction which shows that $z=0$ and completes the proof of the lemma.

It was shown in [10, Proposition 3] that every von Neumann regular right selfinjective ring $R$ satisfies that a countable direct sum of copies of $R_{R}$ is a CSmodule. However, if $R$ is not semisimple, then $R_{R}$ does not have an indecomposable decomposition. In the next theorem, which is an important step towards the proof of the main result, we show more generally that, for modules with a cyclic essential submodule, the above property for the first uncountable cardinal is strong enough to ensure the existence of an indecomposable decomposition.

Theorem 2.2. Let $M$ be a module such that $M^{(J)}$ is a CS-module for some uncountable set $J$ and suppose that $M$ contains a cyclic essential submodule. Then $M$ is a (finite) direct sum of uniform modules.

Proof. Suppose that $M$ is not a direct sum of uniform modules. Since $M$ is a CS-module, this amounts to saying that $M$ is not a direct sum of indecomposable modules and, using [14, Theorem 2.17], we see that $M$ has a local direct summand $\left\{L_{i} \mid i \in J\right\}$ which is not a summand. Thus there exists an infinite countable subset $I$ of $J$ such that $L_{i} \neq 0$ for every $i \in I$ and so $\left\{L_{i} \mid i \in I\right\}$ is a local direct summand of $M$ with $|I|=\aleph_{0}$. Moreover, if $\bigoplus_{I} L_{i}$ is not essential in $M$, then we can take an essential closure $M^{\prime}$ of $\bigoplus_{I} L_{i}$ and write $M=M^{\prime} \oplus N$. Then $\left\{L_{i} \mid i \in I\right\} \cup\{N\}$ is also a countable local direct summand of $M$ and $\left(\bigoplus_{I} L_{i}\right) \oplus N$ is clearly essential in $M$, so that replacing $\left\{L_{i} \mid i \in I\right\}$ by $\left\{L_{i} \mid i \in I\right\} \cup\{N\}$ if necessary, we can assume that $\bigoplus_{I} L_{i}$ is essential in $M$.

Consider now subsets $\mathcal{A}, \mathcal{K}$ of $2^{I}$ which satisfy the conditions stated in Lemma 2.1, so that if $L_{K}$ is an essential closure of $\bigoplus_{K} L_{i}$ in $M$, for each $K \in \mathcal{K}$, then $L_{K} \cap\left(L_{K_{1}}+\cdots+L_{K_{n}}\right)=\bigoplus_{K \cap\left(K_{1} \cup \cdots \cup K_{n}\right)} L_{i}$, provided that $K, K_{1}, \ldots, K_{n} \in \mathcal{K}$ are pairwise distinct. Let $f_{K}: L_{K} \rightarrow \sum_{K \in \mathcal{K}} L_{K}$ be the canonical inclusion, for each $K \in \mathcal{K}$. These inclusions induce an epimorphism

$$
f: \bigoplus_{K \in \mathcal{K}} L_{K} \rightarrow \sum_{K \in \mathcal{K}} L_{K}
$$

Let $L=\bigoplus_{A \in \mathcal{A}} L_{A} \subseteq \bigoplus_{K \in \mathcal{K}} L_{K}$. Then $f(L)=\sum_{A \in \mathcal{A}} L_{A} \subseteq \sum_{K \in \mathcal{K}} L_{K}$. By Lemma 2.1 the sum $\sum_{A \in \mathcal{A}} L_{A}$ is direct and so $\left.f\right|_{L}$ is a monomorphism whose image contains $\bigoplus_{I} L_{i}$ and hence is essential in $\sum_{K \in \mathcal{K}} L_{K}$.

Observe now that, since $|\mathcal{K}|=\aleph_{1}$, it follows from the hypotheses that $\bigoplus_{\mathcal{K}} L_{K}$, being a direct summand of $M^{(\mathcal{K})}$, is a CS-module. Let $X$ be an essential closure of $\operatorname{Ker} f$ in $\bigoplus_{\mathcal{K}} L_{K}$. Then $X$ is a direct summand of $\bigoplus_{\mathcal{K}} L_{K}$ and $X \cap L=0$ because Ker $f \cap L=0$. Now, from Ker $f \subseteq X$ and $X \cap L=0$ it follows that $f(X) \cap f(L)=0$. As $f(L)$ is essential in $\sum_{K \in \mathcal{K}} L_{K}$, we see that $f(X)=0$ and so $\operatorname{Ker} f=X$ is a direct summand of $\bigoplus_{\mathcal{K}} L_{K}$ and $f$ is a split epimorphism. Thus there exists a homomorphism $\varepsilon: \sum_{K \in \mathcal{K}} L_{K} \rightarrow \bigoplus_{\mathcal{K}} L_{K}$ such that $f \circ \varepsilon=1_{\sum_{\mathcal{K}} L_{K}}$.

Consider now a cyclic essential submodule $x R$ of $M$. Then $x R \cap L_{i} \neq 0$ for each $i \in I$ and so there exist elements $r_{i} \in R$ such that $0 \neq x r_{i} \in L_{i}$. Let $Y \subseteq \sum_{K \in \mathcal{K}} L_{K}$ be the submodule generated by the family $\left\{x r_{i}\right\}_{i \in I}$. Because $I$ is countable, $Y$ is 
a countably generated submodule of $\sum_{K \in \mathcal{K}} L_{K}$ and so there exists a countable subset $\mathcal{J} \subseteq \mathcal{K}$ such that $\varepsilon(Y) \subseteq \bigoplus_{K \in \mathcal{J}} L_{K}$. Since $\mathcal{K}$ is uncountable, there exists $K_{0} \in \mathcal{K}-\mathcal{J}$. Let $p_{K_{0}}: M \rightarrow L_{K_{0}}$ be a projection and set $z=p_{K_{0}}(x) \in L_{K_{0}}$. Then, for each $i \in K_{0}$ we have that $0 \neq x r_{i} \in L_{i} \subseteq L_{K_{0}}$ and hence $z r_{i}=p_{K_{0}}(x) r_{i}=$ $p_{K_{0}}\left(x r_{i}\right)=x r_{i} \neq 0$.

Now let $\alpha: \bigoplus_{\mathcal{K}} L_{K} \rightarrow \bigoplus_{\mathcal{J}} L_{K}$ and $\beta: \bigoplus_{\mathcal{J}} L_{K} \rightarrow \bigoplus_{\mathcal{K}} L_{K}$ be the canonical projection and injection, respectively, and consider the homomorphism $\zeta=f \circ \beta \circ$ $\alpha \circ \varepsilon: \sum_{\mathcal{K}} L_{K} \rightarrow \sum_{\mathcal{K}} L_{K}$. Then $\operatorname{Im} \zeta \subseteq \sum_{\mathcal{J}} L_{K}$ and so we have in particular that $\zeta(z) \in \sum_{\mathcal{J}} L_{K}$. Thus there exists a finite set $\left\{K_{1}, \ldots, K_{n}\right\} \subseteq \mathcal{J}$ such that $\zeta(z) \in L_{K_{1}}+\cdots+L_{K_{n}}$. Let $F=K_{0} \cap\left(K_{1} \cup \cdots \cup K_{n}\right)$. Then $F$ is finite because $K_{0} \notin \mathcal{J}$. Since $K_{0}$ is an infinite set, there exists $i_{0} \in K_{0}-F$. For this index we have that $z r_{i_{0}}=x r_{i_{0}} \neq 0$. Observe now that the restriction $\left.\zeta\right|_{Y}$ is just the canonical inclusion of $Y$ in $\sum_{\mathcal{K}} L_{K}$ and so $z r_{i_{0}}=\zeta\left(z r_{i_{0}}\right)$ because $z r_{i_{0}} \in Y$. Thus $z r_{i_{0}}=\zeta\left(z r_{i_{0}}\right) \in L_{K_{0}} \cap\left(L_{K_{1}}+\cdots+L_{K_{n}}\right)=\bigoplus_{F} L_{i}$. Since $z r_{i_{0}} \in L_{i_{0}}$ and $i_{0} \notin F$, we obtain a contradiction which completes the proof.

We are now ready to give our main result. For a module $M$ we will use the category $\sigma[M]$ which is defined as the full subcategory of Mod- $R$ whose objects are all the submodules of $M$-generated modules [19]. $\sigma[M]$ is a Grothendieck category and hence it has injective hulls. The injective objects of $\sigma[M]$ are just the $M$ injective modules. $M$ is called quasi-injective when it is injective in $\sigma[M]$ and $\Sigma$-quasi-injective when every direct sum of copies of $M$ is quasi-injective. The quasi-injective hull of $M$ is precisely the injective hull of $M$ in $\sigma[M]$.

Theorem 2.3. Let $M$ be a $\Sigma$-CS-module. Then $M$ is a direct sum of uniform modules.

Proof. Suppose that $M$ is $\Sigma$-CS and let $\Lambda$ be the set of all local direct summands $\left\{Q_{i}\right\}_{I}$ of $M$ such that each $Q_{i}$ is a uniform module. Clearly $\Lambda \neq \emptyset$ and we can order $\Lambda$ by inclusion, so that it becomes an inductive set and hence, by Zorn's lemma, it contains a maximal element $\left\{Q_{i}\right\}_{I}$. Next we show that $\bigoplus_{I} Q_{i}$ is essential in $M$. Assume that this is not the case and let $L$ be an essential closure of $\bigoplus_{I} Q_{i}$ in $M$. Then $L$ is a direct summand of $M$ and hence we can write $M=L \oplus N$ for some $N \neq 0$. Let $0 \neq x \in N$ and let $N_{x}$ be an essential closure of $x R$ in $N$, which is an essentially finitely generated direct summand of $M$. By Theorem $2.2 N_{x}$ is a direct sum of uniform modules. If $U$ is a nonzero uniform direct summand of $N_{x}$, then it is clear that $\left\{Q_{i}\right\}_{I} \cup\{U\} \in \Lambda$, contradicting the maximality of $\Lambda$. Hence we have that $\bigoplus_{I} Q_{i} \subseteq_{e} M$.

Now, we claim that $M \in \sigma\left[\bigoplus_{I} Q_{i}\right]$. Let $0 \neq x \in M$ and let $L_{x}$ be an essential closure of $x R$ in $M$. Again, by Theorem [2.2, $L_{x}$ is a (finite) direct sum of uniform modules, say $L_{x}=\bigoplus_{j=1}^{n} U_{j}$. Moreover, $U_{j} \cap\left(\bigoplus_{I} Q_{i}\right) \neq 0$ for each $j=1, \ldots, n$, because $\bigoplus_{I} Q_{i}$ is essential in $M$. Let $y_{j} \in U_{j} \cap\left(\bigoplus_{I} Q_{i}\right)$. Then there exists a finite set $I_{j} \subseteq I$ such that $y_{j} R \subseteq \bigoplus_{I_{j}} Q_{i}$. Since $\left\{Q_{i}\right\}_{I}$ is a local direct summand of $M$ we have that $\bigoplus_{I_{j}} Q_{i}$ is a direct summand of $M$ and we can consider a projection $\pi_{j}: M \rightarrow \bigoplus_{I_{j}} Q_{i}$. Then $\left.\pi_{j}\right|_{y_{j} R}$ coincides with the inclusion of $y_{j} R$ in $\bigoplus_{I_{j}} Q_{i}$ and is, therefore, a monomorphism. Since $U_{j}$ is uniform, this implies that $\left.\pi_{j}\right|_{U_{j}}$ is also a monomorphism, so that $U_{j}$ embeds in $\bigoplus_{I} Q_{i}$ and hence $L_{x}$ embeds in a finite direct sum of copies of $\bigoplus_{I} Q_{i}$. Thus $L_{x} \in \sigma\left[\bigoplus_{I} Q_{i}\right]$ and if we pick an essential closure $L_{x}$ of $x R$ for every $x \in M$, we see that also $M \in \sigma\left[\bigoplus_{I} Q_{i}\right]$ because $M$ is generated by the family $\left\{L_{x} \mid x \in M\right\}$. Therefore we have that $\sigma\left[\bigoplus_{I} Q_{i}\right]=\sigma[M]$. 
Now let $Q$ be the quasi-injective hull of $M$, i.e., the injective hull of $M$ in the category $\sigma[M]$. Then $Q$ is also the injective hull of $M$ in the category $\sigma\left[\bigoplus_{I} Q_{i}\right]$ and, because $\bigoplus_{I} Q_{i} \subseteq_{e} M, Q$ is the quasi-injective hull of $\bigoplus_{I} Q_{i}$. Now, $\bigoplus_{I} Q_{i}$ is a $\Sigma$-CS-module because it is a direct summand of the $\Sigma$-CS-module $M^{(I)}$ and hence $Q$ is $\Sigma$-quasi-injective by [1, Corollary 3.20]. But then, again by [1, Corollary 3.20], $M$ is a direct sum of uniform modules.

A decomposition $M=\bigoplus_{I} M_{i}$ is said to complement direct summands (cf. [2] §12]) when, for every direct summand $N$ of $M$, there exists a subset $J \subseteq I$ such that $M=N \oplus\left(\bigoplus_{J} M_{i}\right)$. A module $M$ is said to have the exchange property if, for any index set $I$, whenever $M \oplus N=\bigoplus_{i \in I} X_{i}$ for modules $N$ and $X_{i}$, then $M \oplus N=M \oplus\left(\bigoplus_{i \in I} Y_{i}\right)$ for submodules $Y_{i} \subseteq X_{i}$.

Corollary 2.4. Let $M$ be a $\Sigma$-CS-module. Then $M$ is a direct sum of modules with local endomorphism rings and, furthermore, any such decomposition complements direct summands. In particular, all indecomposable decompositions of $M$ are equivalent and, moreover, $M$ has the exchange property.

Proof. By Theorem 2.3 $M$ is a direct sum of uniform modules which are $\Sigma$-CS and hence $\Sigma$-quasi-injective by [1, Proposition 2.3]. Thus these uniform modules have local endomorphism rings and so the uniqueness of the decomposition follows from Azumaya's theorem [2, Theorem 12.6]. Moreover, the indecomposable decompositions of $M$ complement direct summands, and $M$ has the exchange property by [6. Corollary 3.5].

We can take advantage of Theorem 2.3 to characterize $\Sigma$-CS-modules among CS-modules. In [1, §3] several characterizations of $\Sigma$-CS-modules are given under the additional hypothesis that they are direct sums of uniform modules. Now we know that we can lift this hypothesis from these characterizations and, as a sample result, we have:

Corollary 2.5. Let $M$ be a module. Then the following conditions are equivalent:

(i) $M$ is $\Sigma$-CS.

(ii) $M$ is CS, the quasi-injective hull of $M$ is $\Sigma$-quasi-injective, and $M$ is a direct sum of indecomposable quasi-injective modules.

(iii) $M$ is countably $\Sigma$-CS, and a direct sum of modules with local endomorphism rings.

Proof. (i) $\rightarrow$ (ii) follows from Theorem 2.3, together with [1, Proposition 2.3, Corollary 3.20] and (ii) $\rightarrow$ (i) follows from [1, Theorem 3.9] or [7, Theorem 3.3]. On the other hand, (i) $\rightarrow$ (iii) follows from Corollary 2.4, and (iii) $\rightarrow$ (i) follows from 1. Proposition 3.5].

The next corollary makes precise the difference between $\Sigma$-quasi-injective and $\Sigma$-CS. Recall that a module $M$ is quasi-continuous if it is CS and whenever $M_{1}$ and $M_{2}$ are direct summands of $M$ such that $M_{1} \cap M_{2}=0$, then $M_{1} \oplus M_{2}$ is also a direct summand of $M$.

Corollary 2.6. A module $M$ is $\Sigma$-quasi-injective if and only if it is $\Sigma$-CS and quasi-continuous.

Proof. This follows from Theorem 2.3 and [1, Lemma 4.6]. 
Corollary 2.7. Let $R$ be a ring such that each right $C S$-module is $\Sigma$-CS. Then $R$ is a right noetherian ring.

Proof. By Theorem 2.3 every right CS-module is a direct sum of uniform modules. Then $R$ is right noetherian by [15, Theorem 4].

Corollary 2.7] gives a positive answer to a question of Dung (cf. the comments preceding Proposition 3.9 in [7]). Note, however, that the property that every CS right $R$-module is a direct sum of uniforms is equivalent to $R$ being right noetherian and hence is substantially weaker than having every right CS-module $\Sigma$-CS. In fact, there are (left and right) artinian right serial rings for which not all the right CSmodules are $\Sigma$-CS (cf. 1, Examples 5.13]).

Now we go back to Theorem 2.2 and show how it can be used in certain cases to ensure the existence indecomposable decompositions for CS-modules without the full force of the $\Sigma$-CS hypothesis.

Corollary 2.8. Let $M$ be a countably generated module. If $M^{(I)}$ is a CS-module for some uncountable set $I$, then $M$ is a direct sum of uniform modules.

Proof. By the proof of [9. Lemma 10.1], a countably generated CS-module is a direct sum of modules which contain an essential cyclic submodule. Then the result follows from Theorem 2.2

Observe that from Corollary 2.8 it follows that if $M$ is a projective object of $\sigma[M]$ (also called a $\Sigma$-quasi-projective module) such that $M^{(I)}$ is a CS-module for some uncountable set, then $M$ is a direct sum of uniforms. Indeed, in this case $M$ is a direct summand of a direct sum of finitely generated modules [19, 18.4], and by Kaplansky's theorem [2] Theorem 26.1] $M$ is a direct sum of countably generated modules. This applies, in particular, to the case in which $M$ is a projective module.

Next we show that in the nonsingular case the hypothesis of the preceding corollary is actually equivalent to the $\Sigma$-CS condition. A module $N$ of $\sigma[M]$ is called $M$-singular if $N \cong X / K$ with $X \in \sigma[M]$ and $K$ essential in $X$. $N$ is non- $M$-singular when it has no nonzero $M$-singular submodules.

Corollary 2.9. Let $M$ be a countably generated non- $M$-singular module. Then $M$ is $\Sigma-C S$ if and only if $M^{(I)}$ is CS for some uncountable set $I$.

Proof. If $I$ is an uncountable set such that $M^{(I)}$ is CS, then, by Corollary 2.8, $M$ is a direct sum of uniform modules. Hence $M$ is $\Sigma$-CS by [1, Proposition 3.3].

The preceding corollary gives an affirmative answer to the question posed in [3, Remark 4] by providing the following stronger form of Goodearl's theorem [12] Theorem 2.15]:

Corollary 2.10. If $R$ is a right nonsingular ring such that every $\aleph_{1}$-generated nonsingular right $R$-module is projective, then every nonsingular right $R$-module is projective and hence $R$ is Morita-equivalent to a finite direct product of full upper triangular matrix rings over division rings.

Proof. By the proof of [10, Proposition 1], if every $\aleph_{1}$-generated nonsingular right $R$-module is projective, then $R^{(I)}$ is a CS-module for some uncountable set $I$. Thus $R$ is right $\Sigma$-CS by Corollary 2.9 and hence, again by [10, Proposition 1], every nonsingular right $R$-module is projective. By Goodearl's theorem 12, Theorem $2.15]$ these are precisely the rings which are Morita-equivalent to finite products of full upper triangular matrix rings over division rings. 


\section{REFERENCES}

[1] A.O. Al-attas and N. Vanaja, On $\Sigma$-extending modules, Comm. Algebra 25 (1997), 23652393. MR 98h:16007

[2] F.W. Anderson and K.R. Fuller, Rings and categories of modules, Springer-Verlag, Berlin and New York, 1974. MR 54:5281

[3] J. Clark and N.V. Dung, On the decomposition of nonsingular CS modules, Canadian Math. Bull. 39 (1996), 257-265. MR 97g:16006

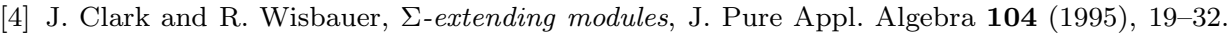
MR 96k:16011

[5] — Polyform and projective $\Sigma$-extending modules, Algebra Colloquium 5 (1998), 391408. CMP 99:11

[6] N.V. Dung, On indecomposable decompositions of CS-modules, J. Australian Math. Soc. 61 (1996), 30-41. MR 97i:16005

[7] _ On indecomposable decompositions of CS-modules II, J. Pure Appl. Algebra 119 (1997), 139-153. MR 98h:16006

[8] Modules with indecomposable decompositions that complement maximal direct summands, J. Algebra 197 (1997), 449-467. MR 98k:16008

[9] N.V. Dung, D.V. Huynh, P.F. Smith, and R. Wisbauer, Extending modules, Longman, Harlow, 1994. MR 96f:16008

[10] N.V. Dung and P.F. Smith, $\Sigma$-CS-modules, Commun. Algebra 22 (1994), 83-93. MR 94m:16003

[11] J.L. Gómez Pardo and P.A. Guil Asensio, On the Goldie dimension of injective modules, Proc. Edinburgh Math. Soc. 41 (1998), 265-275. MR 99e:16027

[12] K.R. Goodearl, Singular torsion and the splitting properties, Mem. Amer. Math. Soc. 124 (1972). MR 49:5090

[13] M. Harada, Factor categories with applications to direct decomposition of modules, Marcel Dekker, New York, 1983. MR 85k:16002

[14] S.H. Mohamed and B.J. Müller, Continuous and discrete modules, Cambridge University Press, Cambridge, 1990. MR 92b:16009

[15] M. Okado, On the decomposition of extending modules, Math. Japonica 29 (1984), 939-941. MR 86k:16013

[16] K. Oshiro, Lifting modules, extending modules and their applications to QF-rings, Hokkaido Math. J. 13 (1984), 310-338. MR 86b:16008a

[17] B. Osofsky, Noninjective cyclic modules, Proc. Amer. Math. Soc. 19 (1968), 1383-1384. MR 38:185

[18] B. Osofsky and P.F. Smith, Cyclic modules whose quotients have all complement submodules direct summands, J. Algebra 139 (1991), 342-354. MR 92f:16030

[19] R. Wisbauer, Foundations of Module and Ring Theory, Gordon and Breach, Reading, 1991.

Departamento de Alxebra, Universidade de Santiago, 15771 Santiago de Compostela, SPAIN

E-mail address: pardo@zmat.usc.es

Departamento de Matemáticas, Universidad de Murcia, 30100 Espinardo (Murcia), SPAIN

E-mail address: paguil@fcu.um.es 